\title{
Ferramentas digitais para a avaliação do processo de aprendizagem: um mapeamento sistemático da literatura
}

\author{
Jaqueline Molon* - IFRS / PPGIE - UFRGS - jaqueline.molon@canoas.ifrs.edu.br \\ Mariano Nicolao - IFRS - mariano.nicolao@canoas.ifrs.edu.br \\ Sérgio Roberto Kieling Franco - PPGIE/PPGEdu - UFRGS - sergio.franco@ufrgs.br
}

Resumo: Considerar a avaliação como fonte para a definição de ações pedagógicas direcionadas às necessidades dos estudantes exige a adoção de estratégias e ferramentas que possam tornar essa práxis possível. Neste artigo é apresentado um Mapeamento Sistemático da Literatura (MSL) realizado com o objetivo de identificar ferramentas digitais existentes para a avaliação da aprendizagem nas perspectivas formativa ou mediadora. A seleção dos artigos se deu por meio do Portal de Periódicos da Capes. A adoção de critérios de inclusão e exclusão resultou na seleção de 26 artigos para síntese e análise. Os resultados do MSL demonstram a existência de algumas ferramentas que avançam neste tema, no entanto se observa que a busca por recursos para a realização de uma avaliação mais individualizada e focada no desenvolvimento cognitivo dos estudantes ainda é um desafio que exige mais pesquisas na área.

Palavras-chave: avaliação formativa, avaliação mediadora, ferramentas digitais, mapeamento sistemático.

\section{Digital tools for the evaluation of the learning process: a systematic literature mapping}

Abstract: Considering evaluation as a source for the definition of pedagogical actions directed to the needs of students requires the adoption of strategies and tools that can make this praxis possible. This article presents a Systematic Literature Mapping (SLM) carried out with the aim of identifying existing digital tools for the assessment of learning from a formative or mediating perspective. The selection of articles took place through the Capes Journal Portal. The adoption of inclusion and exclusion criteria resulted in the selection of 26 articles for synthesis and analysis. The results of the SLM demonstrate the existence of some tools that advance this theme, however it is observed that the search for resources to carry out a more individualized assessment and focused on students' cognitive development is still a challenge that requires more research in the area.

Keywords: formative assessment, mediative assessment, digital tools, systematic mapping.

\section{Introdução}

Investigar o processo de aprendizagem dos estudantes buscando compreender as causas das dificuldades que enfrentam, os tipos de erros cometidos e as estratégias utilizadas na realização das tarefas propostas são fundamentos de uma prática avaliativa mediadora ou formativa (Hoffmann, 2018; Perrenoud, 2007). Um ensino comprometido deve considerar, entre outros aspectos, "sondar a capacidade cognitiva do sujeito da aprendizagem como condição de qualquer prática docente e sondá-la por intermédio de práticas centradas na atividade discente" (Becker, 2012, p. 42).

Nessa concepção de avaliação inclui-se a observação individual de cada aluno considerando seu processo de construção do conhecimento com a finalidade de procurar as razões para as soluções que são apresentadas (Hoffmann, 2018). A avaliação (formativa ou mediadora) centra-se na promoção da aprendizagem e "isso só pode 
acontecer se o professor aprimorar o trabalho pedagógico" (Villas Boas, 2019, p. 19). Dessa forma, a avaliação será considerada, neste trabalho, como oportunidade de aprendizagem, não como fim, mas como processo de construção e de ação pedagógica.

Neste sentido, entende-se que os erros, as dúvidas e os impasses enfrentados pelos estudantes ao longo de todo o processo de ensino e aprendizagem são importantes fontes de informação para a ação pedagógica subsequente. As respostas (corretas ou não) apresentadas pelos estudantes trazem informações ricas acerca das hipóteses levantadas, seus tipos de raciocínio, as formas de representação e esquematização de seu pensamento e as relações lógicas estabelecidas ao longo da resolução de um problema ou na tentativa de compreender um conceito, procedimento ou conteúdo novo.

A avaliação formativa ou mediadora precisa ser capaz de fornecer indícios acerca dos aspectos que estão impossibilitando o estudante de avançar em seu processo de aprendizagem, identificando evidências de percalços durante a construção de conhecimento e, portanto, deve amparar as decisões e ações de ensino (Hoffmann, 2018; Perrenoud; 2007). Logo, a ausência de um recurso específico para a busca por evidências da aprendizagem dos estudantes ou de suas dificuldades acaba se restringindo à realização de provas de diagnóstico ou testes. Essa prática desconstrói a real função da avaliação, culminando em julgamentos do tipo certo ou errado e na obtenção de medida (nota) de desempenho. Encaminhando, assim, a inexata impressão de que a mesma representa, como resultado final, o quanto o aluno aprendeu ou deixou de aprender sem compromisso com o processo.

Para a efetivação do acompanhamento das aprendizagens de cada estudante é importante que se utilize instrumentos adequados que possam otimizar o trabalho docente, uma vez que coletar, armazenar e analisar dados e informações qualitativas acerca do processo de aprendizagem de cada aluno, sem algum recurso digital para tanto, é uma tarefa árdua e muitas vezes inviável. Há estudos que amparam a utilização de recursos tecnológicos nos processos de ensino, na aprendizagem e na avaliação. Venâncio e Lopes (2013), por exemplo, analisaram artigos publicados em eventos (SBIE, WIE e WAvalia) inseridos no Congresso Brasileiro de Informática na Educação (CBIE) que tratavam da avaliação formativa e acompanhamento da aprendizagem através de recursos digitais, considerando o período de 2008 a 2012. Nessa análise, os autores identificaram quinze (15) artigos, cada um descrevendo uma ferramenta utilizada em ambiente de educação à distância, sendo sete (07) utilizados também na educação presencial.

Dos trabalhos analisados Venâncio e Lopes (2013) observaram que apenas dois trabalhos, descrevem ferramentas digitais que consideravam "uma visualização da informação e dos resultados de maneira facilitada para que, diante de grande número de alunos, o professor possa fazer uma análise segura e rápida do processo de aprendizagem individualizado" (Venâncio e Lopes, 2013, p. 140). A primeira dessas ferramentas, denominada de $A I E D$, utilizou mapas conceituais para a regulação de conteúdos didáticos (Oliveira et al., 2010 apud Venâncio e Lopes, 2013). E a segunda foi o SeeAll, um protótipo de um sistema que "organiza e armazena registros sobre vivências de aprendizagem, habilidades, atitudes e competências, retornando aos professores uma visualização em formato de Mapa de Aproveitamento e de Escala de Oportunidades" (Venâncio e Lopes, 2012 apud Venâncio e Lopes, 2013). Com base nisso, Venâncio e Lopes (2013, p. 140) concluem acerca da "carência na área de visualização da informação" diante da necessidade imprescindível de frequentemente visualizar os resultados das aprendizagens no acompanhamento dos alunos. Contudo, até a publicação deste trabalho, nenhum Mapeamento Sistemático da Literatura (MSL) 
que buscasse o levantamento de artigos publicados em periódicos nessa temática foi identificado, o que nos permite nortear e justificar a necessidade do presente estudo.

\section{Material e Métodos}

Este trabalho foi realizado com objetivo de identificar, a partir de artigos publicados em periódicos científicos, as ferramentas digitais (FD) existentes para a avaliação da aprendizagem (AP), na perspectiva formativa ou mediadora. O estudo caracteriza-se como um Mapeamento Sistemático da Literatura (MSL), um tipo de Revisão Sistemática da Literatura (RSL) utilizado para o levantamento do estado da arte, etapa obrigatória na execução de qualquer pesquisa (Dermeval, Coelho e Bittencourt, 2010). O MSL diferencia-se da RSL, principalmente, pelo enfoque dado à revisão. O MSL é utilizado quando se busca respostas amplas à questões de pesquisa do tipo exploratória, ou seja, se deseja obter uma visão geral acerca de algum tópico de pesquisa, enquanto que a RSL busca responder, de modo mais detalhado, questões de pesquisa específicas do tipo causal (Randolph, 2009). Este MSL seguiu o protocolo descrito por Kitchenham e Charters (2007), ilustrado na Figura 1, sendo executado em três fases: planejamento, condução e relatório.
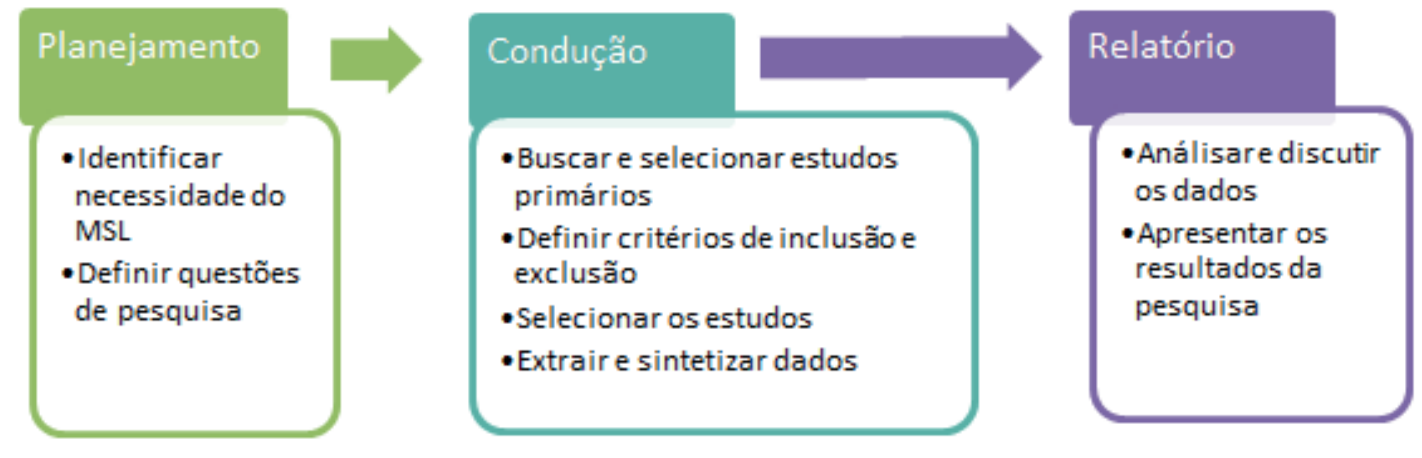

Figura 1. Protocolo de realização do MSL.

Fonte: Elaborada pelos autores baseado em Kitchenham e Charters (2007).

\subsection{Planejamento e Condução}

A necessidade de realização do presente MSL justifica-se pela ausência de um estudo anterior (conforme já exposto) que respondesse à seguinte questão central de pesquisa (QP1): "Que ferramentas ou recursos digitais são utilizados para a avaliação da aprendizagem em contextos educacionais?". A partir dos estudos selecionados buscou-se responder também a outras três questões (Tabela 1):

Tabela 1. Questões de pesquisa secundárias

\begin{tabular}{|l|l|}
\hline QP2 & $\begin{array}{l}\text { Sob que plataformas cada ferramenta foi desenvolvida e como pode ser classificada quanto a sua } \\
\text { concepção tecnológica (aplicativo, plataforma digital, sistema, software, site, etc.)? }\end{array}$ \\
\hline QP3 & De que forma professores e alunos interagem ou utilizam essas ferramentas? \\
\hline QP4 & Como essas ferramentas digitais coletam e disponibilizam os dados para a realização da AP? \\
\hline
\end{tabular}

Fonte: Elaborada pelos autores.

A fim de responder aos questionamentos, definiu-se uma estratégia de busca a partir das palavras-chave "ferramentas digitais" e "avaliação da aprendizagem". Assim, a string de busca foi estruturada: ("digital resources" OR "digital tools" OR "digital tecnolog?" OR "software" OR "digital system" OR "virtual environment") AND ("learning assessment" OR "learning evaluation"). 
A pesquisa foi realizada através do Portal de Periódicos da CAPES $^{1}$ que inclui grande quantidade de bases científicas para consulta entre as quais estão: IEEE Explore, ACM Digital Library, Scielo, ISI Web of Science, Science Direct, SCOPUS, Wiley Online Library, Springer Link (Paz e Cazella, 2018). A busca foi realizada a partir do campo "assunto", utilizando a string de busca apontada, de modo que os termos utilizados na string poderiam aparecer em qualquer parte das publicações. A seleção foi restrita a artigos incluídos em periódicos revisados por pares e publicados no período de 2015 a setembro de 2020. Os procedimentos elencados retornaram 2214 títulos, sendo 104 pré-selecionados para o estudo, dos quais 98 estavam disponíveis para download. A partir da leitura do título, resumo e palavras-chave, aliado aos critérios de inclusão e exclusão, apresentados na Tabela 2, restaram 43 artigos para leitura completa.

Tabela 2. Critérios de inclusão e exclusão utilizados no MSL

\begin{tabular}{|l|l|}
\hline Critérios de Inclusão (CI) & Critérios de Exclusão (CE) \\
\hline Cl1 - Artigo original publicado entre 2015 e set/2020 & CE1 - Artigo de revisão, resumo ou relatório técnico \\
\hline Cl2 - Artigo de periódico revisado por pares & CE2 - Artigo duplicado ou redundantes de mesma autoria \\
\hline $\begin{array}{l}\text { Cl3 - Artigo relacionado ao contexto da pesquisa: AP } \\
\text { em contextos educacionais }\end{array}$ & CE3 - Artigo com pouca aderência ao contexto da pesquisa \\
\hline $\begin{array}{l}\text { Cl4 - Artigo que aborda o uso ou o desenvolvimento } \\
\text { de ferramenta(s) digital(is) para AP. }\end{array}$ & $\begin{array}{l}\text { CE4 - Artigo teórico e de abrangência genérica sobre a } \\
\text { temática AP ou tecnologias. }\end{array}$ \\
\hline $\begin{array}{l}\text { Cl5 - Artigo de acesso livre ou possibilitado através } \\
\text { de convênio institucional com o Portal CAPES }\end{array}$ & $\begin{array}{l}\text { CE5 - Artigo com acesso restrito, apesar de convênio } \\
\text { institucional com o Portal CAPES. }\end{array}$ \\
\hline
\end{tabular}

Fonte: Elaborada pelos autores.

Para ser selecionado, cada artigo deveria cumprir todos os critérios de inclusão, contudo para ser excluído bastava atender a um dos critérios de exclusão. $\mathrm{Na}$ etapa seguinte, de leitura completa, outros critérios de exclusão (Tabela 3) foram utilizados, buscando analisar qualitativamente os estudos pré-selecionados.

Tabela 3. Critérios de exclusão/análise qualitativa

\begin{tabular}{|l|l|}
\hline CE6 & Artigo que não abordava o tópico específico "ferramentas digitais para a avaliação da aprendizagem"; \\
\hline CE7 & $\begin{array}{l}\text { Artigo referente a sistema específico de gerenciamento de informações acadêmicas, não considerando AP } \\
\text { no contexto considerado nesta pesquisa; }\end{array}$ \\
\hline CE8 & Artigo que não trazia características funcionais ou de desenvolvimento da ferramenta digital considerada; \\
\hline CE9 & Artigos sobre FD que não possibilitam acompanhamento qualitativo dos estudantes no processo de AP; \\
\hline
\end{tabular}

Fonte: elaborado pelos autores.

\section{Resultados: análise, síntese e discussão dos artigos do MSL}

Após essa etapa de análise qualitativa, foram selecionados 26 artigos para a realização desse estudo. A Tabela 4 apresenta a síntese dos estudos primários selecionados neste MSL. A primeira coluna responde à QP1, indicando as Ferramentas Digitais (FD) identificadas. A segunda coluna traz informações relacionadas às questões de pesquisa secundárias ${ }^{2}$. O protocolo seguido neste MSL e as referências dos artigos incluídos estão disponíveis no link: https:/url.gratis/ghBTW.

\footnotetext{
1 https://www.periodicos.capes.gov.br/

${ }^{2}$ As questões secundárias envolvem aspectos de uso e desenvolvimento das FD. Porém, por vezes, os artigos selecionados tratavam ou do uso ou do desenvolvimento, de modo que a síntese das informações ( $2^{\mathrm{a}}$ coluna da Tabela 4$)$ refere-se às características das ferramentas presentes nas publicações analisadas.
} 
Tabela 4. Extração e síntese de dados

\begin{tabular}{|c|c|}
\hline Autor (ano) - QP1 & Extração de Dados referentes à QP2, QP3 e QP4 - Características das FD \\
\hline $\begin{array}{l}\text { Concept Maps for } \\
\text { Learning (CMfL) }\end{array}$ & $\begin{array}{l}\text { Site que gera mapas conceituais por meio de um algoritmo de associação e } \\
\text { identificação de familiaridade entre mapas (Pathfinder Scaling Algorithm) que compara } \\
\text { os mapas dos alunos com os de especialistas, fornece feedback visual imediato e } \\
\text { indica material de apoio. O CMfL estrutura os dados para os professores, fornecendo } \\
\text { gráficos visuais e registro das modificações nos mapas dos alunos após feedback } \\
\text { (acompanhamento). }\end{array}$ \\
\hline $\begin{array}{l}\text { 2. Jonsdottir; } \\
\text { Jakobsdottir e } \\
\text { Stefansson (2015) } \\
\text { Sistema Educacional } \\
\text { baseado na Web } \\
\text { adaptável e inteligente }\end{array}$ & $\begin{array}{l}\text { Sistema tutor-Web, desenvolvido em Python. Disponibiliza material (slides em pdf) em } \\
\text { unidades independentes, exercícios de múltipla escolha, fornece feedback (resposta } \\
\text { correta previamente cadastrada) e pontua acertos e tentativas considerando as } \\
\text { respostas dos últimos oito itens respondidos por cada aluno. O aluno responde a cada } \\
\text { item, pode recorrer aos materiais disponíveis a qualquer momento e responder } \\
\text { quantas perguntas quiser, monitorando desempenho e nota e decide quando parar. }\end{array}$ \\
\hline $\begin{array}{l}\text { Rede Ontológica } \\
\text { AONet }\end{array}$ & $\begin{array}{l}\text { Software de avaliações semiautomáticas (questões verdadeiro ou falso, múltipla } \\
\text { escolha, mapa conceitual, exercício, atividade de redação, etc.) com três domínios } \\
\text { integrados: tópicos do curso, recursos educacionais e avaliação. A AONet pode } \\
\text { incorporar outras ontologias. A ontologia da avaliação tem quatro perfis de uso (autor, } \\
\text { aluno, professor e gestor) e modela a maioria dos instrumentos avaliativos usados. }\end{array}$ \\
\hline $\begin{array}{l}\text { Sistema de Perfil do } \\
\text { Aluno (SPA) }\end{array}$ & $\begin{array}{l}\text { Sistema que monitora, em tempo real, ações dos alunos em ambientes de } \\
\text { aprendizagem para automatizar a construção de perfis de alunos. Envolve análise } \\
\text { multidimensional. O SPA monitora e mede, por meio de algoritmos, a habilidade } \\
\text { cognitiva (memória, concentração, percepção) e condição de aprendizagem. A análise } \\
\text { das características dos alunos e constituição do SPA considera também informações } \\
\text { de avaliações fornecidas pelos professores e propõe direcionar atividades com a } \\
\text { identificação do perfil do aluno. }\end{array}$ \\
\hline $\begin{array}{l}\text { 5. Santos; Cook e } \\
\text { Hernández-Leo (2015) } \\
\text { m-AssIST }\end{array}$ & $\begin{array}{l}\text { O sistema M-AssIST (mobile-Assessment Interaction Scaffolding Temporal Model) } \\
\text { captura características das atividades e interações dos alunos na plataforma. Com } \\
\text { isso, o m-AssIST cria cenários para apoiar as interações em contexto avaliativo (entre } \\
\text { alunos, com o ambiente, com a atividade de avaliação ou informações de suporte). As } \\
\text { interações podem ser monitoradas e avaliadas automaticamente, mas algumas só } \\
\text { consideram as respostas fornecidas pelos próprios alunos, sendo este um aspecto } \\
\text { negativo apontado. }\end{array}$ \\
\hline $\begin{array}{l}\text { Student Response } \\
\text { Systems (SRS) }\end{array}$ & $\begin{array}{l}\text { Consiste em cartões eletrônicos que transmitem respostas a um receptor conectado } \\
\text { ao notebook do professor (via Wi-fi, radiofrequência ou infravermelho). Envolve três } \\
\text { atividades: instruções e perguntas, exibição de respostas, gerenciamento de dados e } \\
\text { análise de estudos. O professor apresenta conceitos e materiais, desenvolve slides de } \\
\text { perguntas (usando a tecnologia Turning Point) e solicita feedback aos alunos. Os } \\
\text { alunos usam transmissores remotos que enviam sinais para o computador do } \\
\text { professor. O SRS compila os dados e constrói um gráfico das respostas, permitindo a } \\
\text { retomada das dúvidas antes de encerrar a apresentação daquele tópico. }\end{array}$ \\
\hline $\begin{array}{l}\text { Sistema de } \\
\text { aprendizagem } \\
\text { baseado em CBGLA }\end{array}$ & $\begin{array}{l}\text { Sistema pautado em um algoritmo de aprendizagem guiada baseado em } \\
\text { competência" (CBGLA). O algoritmo gera caminhos de aprendizagem personalizados } \\
\text { de acordo com habilidades iniciais de cada aluno, avaliadas através de um pré-teste e } \\
\text { da atribuição de uma pontuação. O algoritmo de classificação, do tipo bolha, compara } \\
\text { os objetivos de aprendizagem com a pontuação obtida a cada módulo e marca os } \\
\text { módulos em que o aluno já atingiu os padrões de competência. Os módulos dos } \\
\text { cursos são organizados em ordem crescente de proficiência. O sistema orienta o } \\
\text { aluno progressivamente e elimina os módulos cujos requisitos tenham sido atingidos. }\end{array}$ \\
\hline $\begin{array}{l}\text { Análise de arquivos } \\
\text { de log na SPC em } \\
\text { avaliação assistido } \\
\text { por computador }\end{array}$ & $\begin{array}{l}\text { O estudo investigou a relação entre comportamentos dos alunos e desempenho na } \\
\text { solução de problemas complexos (SPC), em avaliações assistidas por computador, } \\
\text { através de registros de log e revelou que quanto maior a necessidade de ações } \\
\text { exploratórias para medir uma habilidade específica, mais longas serão as sequências } \\
\text { comportamentais expressas por essas ações, resultando em arquivos de log mais } \\
\text { extensos e com maior quantidade de informações. Nove indicadores dicotômicos para } \\
\text { modelar a aquisição de conhecimento nas tarefas foram desenvolvidos. Observou-se } \\
\text { que: alunos que analisavam os problemas antes de executar ações exploratórias } \\
\text { tiveram melhor desempenho na SPC em relação àqueles que realizavam grande } \\
\text { frequência de intervenções; o baixo desempenho pode ser associado ao tempo (muito } \\
\text { pouco ou muito tempo na tarefa); a relação tempo/desempenho não é linear e } \\
\text { depende de características da tarefa, da dificuldade e nível de proficiência do aluno. }\end{array}$ \\
\hline $\begin{array}{l}\text { 9. Nacheva-Skopalik e } \\
\text { Green (2016) } \\
\text { Ferramentas WIDGaT } \\
\text { para autoria de }\end{array}$ & $\begin{array}{l}\text { Protótipos de aplicações pequenas (widgets) que, ao serem incorporadas a um em um } \\
\text { sistema inteligente e adaptável de e-learning, fornecem acessibilidade para avaliação } \\
\text { automatizada. Em cada caso de uso e cada avaliação, widgets de avaliação eletrônica } \\
\text { criadas com são ferramentas de autoria WIDGaT são incorporadas no ambiente e }\end{array}$ \\
\hline
\end{tabular}




\begin{tabular}{|c|c|}
\hline $\begin{array}{l}\text { aplicações focadas na } \\
\text { avaliação inclusiva }\end{array}$ & $\begin{array}{l}\text { permitem adaptações escolhidas pelo tutor/professor, pelo aluno ou definição de } \\
\text { forma automática, conforme necessidades educacionais específicas dos estudantes. }\end{array}$ \\
\hline $\begin{array}{l}\text { 10. Ramírez-Noriega, } \\
\text { Juárez-Ramíreza e } \\
\text { Martínez-Ramírez } \\
\text { (2016) } \\
\text { Avaliação usando } \\
\text { Redes Bayesianas em } \\
\text { um STI }\end{array}$ & $\begin{array}{l}\text { Módulo de avaliação incorporado a um Sistema de Tutoria Inteligente (STI) para inferir } \\
\text { o nível de conhecimento dos alunos utilizando redes bayesianas. A ideia principal é } \\
\text { que o aluno tenha acesso às aulas para estudar um tópico, faça provas, exercícios e } \\
\text { atividades associadas (questões de múltipla escolha com correção automática e } \\
\text { cômputo de acertos e erros). A interação do aluno com o STI gera evidências quanto } \\
\text { ao seu conhecimento que passam a integrar a estrutura da rede, possibilitando inferir } \\
\text { conhecimento do aluno sobre conceitos, tópicos ou unidades específicas. Cada } \\
\text { pergunta é escolhida pelo STI com base na resposta anterior, nas relações entre } \\
\text { conceitos e em técnicas de inferência e probabilidade em redes bayesianas. }\end{array}$ \\
\hline $\begin{array}{l}\text { 11. Steif; Fu e Zara } \\
\text { (2016) } \\
\text { Tutor Cognitivo } \\
\text { Inteligente }\end{array}$ & $\begin{array}{l}\text { Tutor cognitivo de suporte para a resolução de problemas de treliça, comuns na } \\
\text { engenharia, que possuem múltiplos caminhos de resolução. O aluno resolve os } \\
\text { problemas no computador e, a cada ação executada, o tutor identifica relações com } \\
\text { uma lista de habilidades ou componentes de conhecimento pré-estabelecida e fornece } \\
\text { feedback através de algoritmos de execução das etapas de resolução desse tipo de } \\
\text { problema. Possui interface gráfica do usuário (permite interações e registros), } \\
\text { algoritmos (julgar as ações) e conjecturas (analisar os registros inseridos pelo aluno). }\end{array}$ \\
\hline $\begin{array}{l}\text { 12. Bradac e Walak } \\
\text { (2017) } \\
\text { Sistema de e-learning } \\
\text { adaptativo para o } \\
\text { ensino de línguas }\end{array}$ & $\begin{array}{l}\text { O sistema usa processamento de informações e detecta a preferência sensorial do } \\
\text { usuário (audição, visão, leitura, etc.) ao usar o ambiente, criando variantes } \\
\text { personalizadas (não usa modelos de aluno para agrupar estudantes). A partir de um } \\
\text { questionário inicial, testes de conhecimento (múltipla escolha com avaliação } \\
\text { automática), análise do tempo e peso da resposta dentro de cada categoria avaliada e } \\
\text { importância da categoria para a sequência de estudos, usa critérios de entrada (if) e } \\
\text { saída (then) para avaliar o conhecimento do aluno e criar plano de estudo adaptado. }\end{array}$ \\
\hline $\begin{array}{l}\text { Modelo de Aluno e } \\
\text { Modelo de Evidências }\end{array}$ & $\begin{array}{l}\text { Este artigo demonstra uma abordagem para a identificação de evidências e } \\
\text { agregação de dados do fluxo de atividades para a criação de Modelo de Aluno. As } \\
\text { evidências são obtidas em dois ambientes digitais diferentes, de respostas a itens de } \\
\text { avaliação tradicionais e de classificações baseadas em observações de atividades } \\
\text { pessoais não digitais, todos alinhados para fazer inferências sobre o domínio do } \\
\text { conhecimento dos alunos ao longo da progressão da aprendizagem. Um modelo de } \\
\text { redes bayesianas (BN), composto por duas partes (modelo de aluno e modelo de } \\
\text { evidência) foi usado para agregar evidências das múltiplas fontes de avaliação. }\end{array}$ \\
\hline $\begin{array}{l}\text { 14. Ferreira et al. } \\
\text { (2017) }\end{array}$ & $\begin{array}{l}\text { O sistema BOCA é um sistema de correção automática de programação usando } \\
\text { entradas e saídas predefinidas, que serve como um repositório das soluções dos } \\
\text { alunos e onde o feedback sobre a correção do programa é fornecido (os dados de } \\
\text { entrada são usados para verificar se a saída do programa é idêntica à saída esperada } \\
\text { para aquela entrada. No entanto, aspectos como clareza do programa e design não } \\
\text { podem ser verificados por sistemas de avaliação automática de programação. O } \\
\text { estudo indica a necessidade de melhora dos processos de avaliação do desempenho } \\
\text { do aluno com a adoção de práticas, ambientes e meios de avaliação que contribuam } \\
\text { com a construção do conhecimento, permitam interações entre os alunos e } \\
\text { professores e identificação do que deve ser melhorado, não só o julgamento do } \\
\text { programa final. }\end{array}$ \\
\hline $\begin{array}{l}\text { 15. Giabbanelli e Tawfik } \\
\text { (2017) } \\
\text { Incremental Thesaurus } \\
\text { for Assessing Causal } \\
\text { Maps (ITACM) }\end{array}$ & $\begin{array}{l}\text { Software que auxilia na avaliação de mapas causais, que indicam a relação entre dois } \\
\text { conceitos. A avaliação automática desses mapas enfrenta desafios, já que termos } \\
\text { diferentes podem indicar o mesmo conceito. O sistema é incremental: um avaliador } \\
\text { compara o mapa causal do aluno e do especialista e incorpora os termos similares } \\
\text { identificados e, na medida em que mais mapas são avaliados, mais automatizada fica } \\
\text { a avaliação devido ao acréscimo de termos no ITACM. }\end{array}$ \\
\hline $\begin{array}{l}\text { 16. Murchan \& Oldham } \\
\text { (2017) } \\
\text { Avaliação Baseada } \\
\text { em Computador } \\
\text { (CBA) }\end{array}$ & $\begin{array}{l}\text { Os autores tratam da Avaliação Assistida por Computador (AAC) como apoio ao } \\
\text { ensino e aprendizagem de matemática descrevendo uma Avaliação Baseada em } \\
\text { Computador (CBA) projetada para as séries intermediárias das escolas primárias } \\
\text { irlandesas. O teste contém uma variedade de formatos de itens, principalmente de } \\
\text { natureza aberta. O sistema avalia as respostas, atribui pontuação automática e usa } \\
\text { técnicas de análise de erros para classificar os tipos de erros cometidos pelos alunos } \\
\text { e para fornecer informações úteis sobre os seus desempenhos. }\end{array}$ \\
\hline $\begin{array}{l}\text { 17. Saputra, Budiyanto } \\
\text { e Hatta (2017) } \\
\text { Software de avaliação } \\
\text { de competências } \\
\text { baseado na Web }\end{array}$ & $\begin{array}{l}\text { Desenvolvido com base no modelo proposto por Pressman (2010) em PHP com } \\
\text { banco MySQL. Possui três usuários: administrador (gerencia o sistema), professor } \\
\text { (gerenciar o banco de perguntas, itens dos testes e resultados) e estudantes (que } \\
\text { fazem o teste). Recursos: correção automática dos testes; adição de multimídias, uso } \\
\text { de dados e fórmulas do Microsoft Excel para atribuição de pontuação automática. }\end{array}$ \\
\hline $\begin{array}{l}\text { 18. Amasha et al. (2018) } \\
\text { Formulário de } \\
\text { Sincronização de } \\
\text { Aprendizado e }\end{array}$ & $\begin{array}{l}\text { O estudo avaliou a sincronização de conteúdo com avaliação imediata através do } \\
\text { OLASF e o desempenho dos alunos. O Nearpod é um aplicativo Web utilizado como } \\
\text { plataforma on-line para a disponibilização de materiais, realização de testes e } \\
\text { fornecimento de orientações aos alunos. Os resultados mostram que a avaliação on- } \\
\text { line para avaliação formativa, além de fornecer dados ao professor sobre o }\end{array}$ \\
\hline
\end{tabular}




\begin{tabular}{|c|c|}
\hline $\begin{array}{l}\text { avaliação imediata on- } \\
\text { line (OLASF) }\end{array}$ & desenvolvimento do curso, possibilita que os alunos aprendam com seus erros. \\
\hline $\begin{array}{l}\text { 19. Laakso; Kaila e } \\
\text { Rajala (2018) }\end{array}$ & $\begin{array}{l}\text { Ferramenta colaborativa web que agrega atividades de aprendizagem e avaliação, } \\
\text { com recursos compartilháveis entre os professores possibilitando adaptações e reuso. } \\
\text { Os alunos através de wikis ou recursos semelhantes podem resolver em conjunto } \\
\text { exercícios através de uma interface de compartilhamento de conta. Os alunos podem } \\
\text { enviar respostas de qualquer lugar a qualquer tempo e receber o feedback imediato. } \\
\text { Vários tipos de exercícios são possíveis (preenchimento de lacunas, perguntas de } \\
\text { múltipla-escolha, respostas curtas, quebra-cabeças, apontamento de informações ou } \\
\text { elementos em figuras, entre outros), além de outros que podem ser projetados } \\
\text { diretamente na ferramenta. Permite envio de respostas longas, ensaios e textos, no } \\
\text { entanto, esses precisam ser corrigidos manualmente. O feedback possibilita fornecer } \\
\text { detalhes de cada tipo de exercício para ajuste de respostas incorretas ou exemplos. }\end{array}$ \\
\hline 20. Yago et al. (2018) & $\begin{array}{l}\text { Modelo de Aluno Baseado em Rede de Ontologia para Ambientes de Aprendizagem } \\
\text { Múltipla (ON-SMMILE) que combina informações relacionadas ao estudante e seu } \\
\text { estado de conhecimento, avaliações e diferentes tipos de objetivos, unidades de } \\
\text { aprendizagem e recursos de informação de suporte em AVA inteligente. É adaptativo, } \\
\text { flexível e voltado ao acompanhamento do progresso dos alunos com o objetivo de } \\
\text { orientá-los. Desenvolvido em módulos seguindo princípios de reusabilidade (aplicação } \\
\text { a diferentes domínios) e extensibilidade (adaptação a novos ambientes ou } \\
\text { tecnologias), além de aplicar diversas técnicas de processamento de dados. }\end{array}$ \\
\hline $\begin{array}{l}\text { 21. Gamage et al. } \\
\text { (2019) } \\
\text { Quizzes Moodle e } \\
\text { análise psicométrica } \\
\text { (índices de facilidade } \\
\text { e discriminação das } \\
\text { questões) }\end{array}$ & $\begin{array}{l}\text { Questionários elaborados através do ambiente Moodle (Quizzes Moodle) foram } \\
\text { elaborados e, a partir da incorporação de análise psicométrica com base em dois } \\
\text { índices (Índice de Facilidade (FI) e Índice de discriminação (DI) das questões) as } \\
\text { perguntas foram adequadas para avaliar e distinguir os níveis de conhecimento dos } \\
\text { alunos. Os índices FI e DI são recursos estatísticos úteis para montar conjuntos de } \\
\text { perguntas que podem tornar as avaliações mais autônomas e que podem ser } \\
\text { incorporados em outros ambientes que possibilitam a criação de questionários, além } \\
\text { do Moodle. Através do índice Fl, entendido como nível de dificuldade das questões, } \\
\text { do índice de discriminação (DI) da questão e da combinação dos resultados de FI e DI } \\
\text { foi possível separar alunos com diferentes níveis de conhecimento. }\end{array}$ \\
\hline $\begin{array}{l}\text { 22. Rao CH e Saha } \\
(2019) \\
\text { RemedialTutor } \\
\text { System }\end{array}$ & $\begin{array}{l}\text { Plataforma de aprendizado assistido por computador com foco em auxiliar alunos com } \\
\text { baixo desempenho em exames. O professor insere um texto no sistema, o } \\
\text { RemedialTutor analisa o texto e executa tarefas conforme solicitado pelo aluno: } \\
\text { fornece significado de palavras desconhecidas; seleciona automaticamente possíveis } \\
\text { perguntas a partir do texto inserido; fornece resumo do conteúdo; realiza exames, } \\
\text { testes experimentais para aumentar a confiança dos alunos; avalia automaticamente } \\
\text { as respostas dos alunos e identifica tópicos que o aluno precisa revisar, funcionando } \\
\text { como um tutor corretivo. }\end{array}$ \\
\hline $\begin{array}{l}\text { Estrutura baseada em } \\
\text { ontologias para E- } \\
\text { learning }\end{array}$ & $\begin{array}{l}\text { Uma estrutura que explora benefícios da Web } 3.0 \text { por meio de diferentes ontologias: } \\
\text { modelos de ontologia de curso e ontologias de avaliação para modelar questionários e } \\
\text { exercícios. Incorporada num sistema E-Learning é capaz de traçar o perfil dos alunos, } \\
\text { categorizar os alunos com base em perfis, fazer recomendações de conteúdo } \\
\text { personalizado e realizar avaliação de conteúdo. Um modelo matemático foi proposto } \\
\text { para a categorização do aluno usando técnicas de aprendizado de máquina } \\
\text { (raciocínio baseado em casos e redes neurais). Os conteúdos da aprendizagem foram } \\
\text { anotados através de Course Ontology com base em três cursos modelados para os } \\
\text { alunos. Atributos como desempenho acadêmico, estilo de aprendizagem, aptidão, } \\
\text { conhecimento prévio e desempenho de longo prazo durante o curso são considerados } \\
\text { ao oferecer os conteúdos de aprendizagem personalizados. Os perfis do aluno são } \\
\text { mantidos em Learner Ontology como classes de ontologia, propriedades e indivíduos. } \\
\text { A fim de categorizar os perfis dos alunos, além do uso de Lógica Fuzzy, combinando } \\
\text { técnicas de aprendizado de máquina (Case Based Reasoning e Redes Neurais } \\
\text { Artificiais) foi proposta: a LCHAIT (Learner Categorization with Hybrid of Artificial } \\
\text { Intelligence Techniques). }\end{array}$ \\
\hline $\begin{array}{l}\text { Game-enhanced } \\
\text { scenario-based } \\
\text { assessment }\end{array}$ & $\begin{array}{l}\text { O artigo descreve um ambiente para avaliação formativa capaz de reunir evidências } \\
\text { sobre as habilidades de argumentação de alunos em cenários aprimorados por jogos. } \\
\text { O jogo (Seeball) inclui uma série de atividades de raciocínio argumentativo projetadas } \\
\text { em torno de progressões de aprendizagem que servem como uma estrutura para } \\
\text { determinar as habilidades, níveis e a sequência de atividades. Essa estrutura } \\
\text { possibilita a coleta de evidências sobre as habilidades de argumentação dos alunos e } \\
\text { a criação de cenários favoráveis ao desenvolvimento da argumentação. Os autores } \\
\text { defendem que em ambientes de avaliação baseados em jogos, dados são reunidos } \\
\text { não apenas por meio das respostas dos alunos às perguntas e solicitações, mas } \\
\text { também por meio de arquivos de log de suas interações dentro das atividades do jogo } \\
\text { que podem fornecer detalhes sobre os processos cognitivos e destacam a } \\
\text { necessidade de mais pesquisas na área para aprimorar as técnicas de avaliação. }\end{array}$ \\
\hline
\end{tabular}




\begin{tabular}{|l|l|}
\hline 25. Cubric e Tosic & $\begin{array}{l}\text { Onto2MCQ é uma ferramenta baseada em ontologias que gera rapidamente questões } \\
\text { (2020) }\end{array}$ \\
de múltipla escolha para avaliações assistidas por computador. Trata-se de aplicativo \\
web usando código aberto e ferramentas de desenvolvimento gratuitas, como Java, \\
PHP, UserCake, MySQ, Apache Jena, Apache PDFBox. Oferece um sistema \\
autônomo totalmente funcional para a geração das questões e distratores (alternativas \\
incorretas). A partir de modelos, questões de diferentes níveis de conhecimento e \\
dificuldade são geradas, considerando a taxonomia de Bloom e empregando \\
estratégias de seleção de distratores. A ferramenta foi avaliada usando critérios como \\
qualidade, dificuldade, validade e utilidade das questões geradas.
\end{tabular}

Fonte: Elaborada pelos autores.

De modo geral conforme as informações sintetizadas na Tabela 4 foi possível averiguar que existem diferentes ferramentas e recursos tecnológicos para a avaliação da aprendizagem (QP1) e que a temática é bastante relevante, tendo em vista o grande número de estudos retornados no Portal da Capes a partir da string de busca utilizada (2214 artigos), bem como o quantitativo remanescente após a aplicação dos critérios de inclusão e exclusão (26 artigos).

Sob diferentes plataformas de desenvolvimento e linguagens de programação (QP2) essas ferramentas digitais são de tipos variados ${ }^{3}$ : sistemas de gerenciamento de conteúdo de aprendizagem (artigos $2 ; 4 ; 5 ; 6 ; 7 ; 12 ; 18 ; 19$ ); sistemas de tutoria inteligente ou tutores cognitivos (artigos $2 ; 10 ; 11 ; 20 ; 22$ ); modelos de aluno e construção de perfis (artigos 4; 13; 20; 23); mapas conceituais/causais (artigos; 1; 3; 15), ferramentas que usam estratégias de comparação de material elaborado por especialistas, contidas em bases de conhecimento ou relacionadas em listas de habilidades ou componentes de conhecimento pré-definidos para avaliar as produções e o desempenho dos alunos (artigos $1 ; 11 ; 13 ; 14 ; 15 ; 26$ ), ferramentas baseadas em testes ou análise de desempenho dos estudantes nas atividades propostas (artigos 4; 6; 7; $8 ; 12 ; 13 ; 16 ; 17 ; 18 ; 20 ; 21 ; 22 ; 23 ; 25)$, algumas recorrendo ainda a algoritmos de classificação ou categorização dos alunos de acordo com conhecimentos e habilidades identificadas (artigos $4 ; 7 ; 12 ; 13 ; 20 ; 21 ; 23$ ).

Com frequência, as ferramentas identificadas incluíam recomendações para a sequência de estudos, baseadas em progressões de aprendizagem ou indicavam tópicos de revisão (artigos $4 ; 7 ; 10 ; 12 ; 18 ; 19 ; 20 ; 22 ; 23 ; 24$ ). Outras, ainda, permitiam a sua utilização por professores e alunos também como repositório de materiais de apoio ou material de uso nas próprias aulas (artigos $2 ; 3 ; 5 ; 6 ; 10 ; 17 ; 18 ; 20 ; 22 ; 23$ ).

Observou-se, contudo, uma grande procura por estratégias estratégias avaliativas centradas na solução de questões fechadas (QP3), de múltipla-escolha ou respostas curtas (artigos $2 ; 3 ; 6 ; 10 ; 17 ; 18 ; 21 ; 23 ; 25$ ) e com atribuição de pontuação de forma automática (artigos $2 ; 6 ; 10 ; 14 ; 16 ; 17 ; 18 ; 23$ ). Algumas ferramentas associavam

\footnotetext{
${ }^{3}$ Algumas ferramentas se enquadram em mais de uma categoria, em função de características funcionais.
} 
técnicas de acompanhamento como o monitoramento das interações e ações dos alunos durante a navegação (artigos $4 ; 5 ; 8 ; 9 ; 12 ; 13 ; 20 ; 22 ; 23 ; 24$ ) utilizando registros de $\log$, por exemplo (artigos 8 e 24) ou gráficos, estatísticas, etc. (artigos $1 ; 6 ; 17 ; 26$ ).

Também foram identificados esforços para a implementação de outros formatos avaliativos como a avaliação automática de respostas longas, textos, mapas conceituais, resoluções de problemas complexos, adaptações de avaliações à necessidades específicas, identificação de preferências dos usuários, utilização de ferramentas colaborativas (artigos $1 ; 5 ; 8 ; 9 ; 11 ; 12 ; 14 ; 15 ; 16 ; 19 ; 23 ; 24 ; 26$ ). Entre os recursos utilizados ou incorporados nas aplicações encontradas, observou-se a adoção de ontologias (artigos 3; 20; 23; 25; 26), redes bayesianas (artigos 10 e 13); regras if/them (estruturas condicionais) e variantes personalizadas (artigo 12), lógica Fuzzy (artigo 23); técnicas de processamento de dados e informações (artigos 12 e 20); aprendizado de máquina (artigo 23); redes semânticas (artigo 26); criação de cenários (artigos 5 e 24). Com isso, foi possível notar que ao considerar mais elementos para a realização da avaliação e acompanhamento dos estudantes, aumenta-se o volume de informações armazenadas e, consequentemente, as possibilidades de obtenção de evidências dos processos de aprendizagem dos estudantes e de suas dificuldades (QP4).

Constatou-se, ainda, uma preocupação constante quanto à necessidade de fornecer feedback aos estudantes como estratégia para promover melhoria em seus desempenhos ou notas (artigos $1 ; 2 ; 6 ; 11 ; 14 ; 16 ; 19 ; 20 ; 22$ ). Além disso, a atenção para a possibilidade de adaptação dessas ferramentas às diferentes plataformas também está presente. Alguns recursos são do tipo aplicativo $W e b$, que requerem apenas um servidor, um banco de dados para armazenamento de informações e acesso a internet para utilização, sendo uma vantagem observada em relação aos que dependem de algum tipo de software ou plugin específico. Por fim, há indicações, também, de estudos futuros para desenvolvimento de adaptações nas ferramentas existentes a fim de possibilitar acesso através de dispositivos móveis, em função das características de mobilidade, usabilidade e acessibilidade que esses aparelhos oferecem.

\section{Considerações Finais}

A avaliação precisa estar alinhada aos processos de ensino e de aprendizagem, por isso foram adotadas as perspectivas formativa e mediadora nesse estudo. $\mathrm{O}$ resultado de toda avaliação deve ser fonte para a definição das ações pedagógicas subsequentes, possibilitando direcionamentos às necessidades de aprendizado de cada estudante de acordo com o seu estado de conhecimento. Contudo, por vezes, estratégias de avaliação formativa ou mediadora se confundem com outras, como a somativa. Ao longo da realização deste MSL foram encontrados artigos que faziam referência à avaliação formativa, porém a prática descrita se centrava em testes de conhecimento e na atribuição de nota do desempenho obtido no teste realizado on-line. Nesses casos, percebe-se que há uma transposição da prova escrita para o computador (ou recurso similar), que muitas vezes não está alinhada ao compromisso de avaliação enquanto acompanhamento contínuo, mas como verificação de desempenho.

Os resultados apontam ferramentas existentes, mas indicam que a busca por recursos para a realização de uma avaliação mais individualizada e focada no desenvolvimento cognitivo dos estudantes ainda é um desafio que exige esforços de mais pesquisas na área. Os desafios impostos pelos tempos atuais exigem a busca de alternativas para o acompanhamento da aprendizagem dos estudantes, para além das clássicas provas. Durante o ensino remoto emergencial, prática adotada no período de 
enfrentamento à pandemia de Covid-19, por exemplo, essa necessidade ficou explícita e exigiu rápidas adaptações das ações pedagógicas, incluindo as formas de avaliação.

Por fim, cabe salientar que este estudo possui limitações, as quais são comuns na realização de MSL. Uma delas se encontra na possibilidade de ter desconsiderado ou não obtido estudos ou dados relevantes para a temática em função da necessidade da adoção de critérios de seleção e refinamento da busca. Além disso, embora o Portal da Capes contenha uma grande quantidade de bases e periódicos indexados, ainda existem outras fontes que podem complementar os dados levantados, as quais no entanto precisam ser consultadas manualmente. Pode-se, ainda, retomar estudos já realizados que buscaram por ferramentas a partir de publicações em conferências e eventos de educação em computação e informática na educação, como alguns exemplos citados na introdução deste artigo, os quais por sua vez podem ser atualizados. Assim, como trabalho futuro, espera-se retomar essa pesquisa ampliando sua abrangência.

\section{Agradecimentos}

*A autora agradece pelo apoio e fomento para qualificação recebidos do Instituto Federal de Educação, Ciência e Tecnologia do Rio Grande do Sul (IFRS).

\section{Referências Bibliográficas}

BECKER, F. Educação e Construção do Conhecimento.Porto Alegre: Penso, 2012.

DERMEVAL, D.; COELHO, J. A. P. de M.; BITTENCOURT, Ig I. Mapeamento Sistemático e Revisão Sistemática da Literatura em Informática na Educação. In: JAQUES, P. A.; SIQUEIRA; S.; BITTENCOURT, Ig; PIMENTEL, M. (Org.) Metodologia de Pesquisa Científica em Informática na Educação: Abordagem Quantitativa. Porto Alegre: SBC, 2020. v. 2. Disponível em: $<\underline{\text { https://metodologia.ceie- }}$ br.org/livro-2>.

HOFFMANN, J. Avaliação mediadora: uma prática em construção da pré-escola à universidade. Porto Alegre: Mediação, 2018.

KITCHENHAM, B.; CHARTERS, S. Guidelines for performing Systematic

Literature Reviews in Software Engineering. Technical Report EBSE 2007-001, Departament of Computer Science Keele University, Keele, 2007.

PAZ, F. J.; CAZELLA, S. C. Integrando Sistemas de Recomendação com Mineração de Dados Educacionais e Learning Analytics: Uma revisão sistemática da Literatura. Renote, v.16, n.1, p. 1-10, 2018.

PERRENOUD, P. Avaliação: da excelência à regulação das aprendizagens: entre duas lógicas. Porto Alegre: Artmed, 2007.

RANDOLPH, J. J. A guide to writing the dissertation literature review. Practical Assessment, Research \& Evaluation, v. 14, n. 13, p. 1-13, 2009.

VENÂNCIO, V.; LOPES, R.; Avaliação da Aprendizagem em Sistemas Interativos: uma revisão comparativa focada no SBIE, WIE e WAvalia. In: Anais do II Congresso Brasileiro de Informática na Educação (CBIE 2013), 2013, p.134-143.

VILLAS BOAS, B. Compreendendo a avaliação formativa. In: VILLAS BOAS, Benigna (org.). Avaliação formativa: Práticas inovadoras. Papirus Editora, 2019, p. 13-42. 